\title{
A study on the mechanism of agonists in regulating transcriptional level of pIgR in salivary gland epithelial cells
}

\author{
LI HUANG, CHUANKONG SUN, RUOBING PENG and ZHIMING LIU
}

Department of Stomatology, Renmin Hospital of Wuhan University, Wuhan, Hubei 430060, P.R. China

Received January 1, 2018; Accepted June 25, 2018

DOI: $10.3892 /$ etm.2018.6792

\begin{abstract}
The aim of the present study was to explore the mechanism of agonists in regulating transcriptional level of polymeric immunoglobulin receptor (pIgR) in salivary gland epithelial cells, thus revealing the defense effect of salivary immune on bacteria in the oral cavity. Sixty patients with oral bacterial infection and 70 patients suffering from oral diseases without bacterial infection were selected randomly from patients in Renmin Hospital of Wuhan University from April 2015 to April 2017. Ribonucleic acid (RNA) was extracted from salivary gland epithelial cells of all patients. Fluorescent quantitative polymerase chain reaction (FQ-PCR) and western blotting methods were adopted to detect and compare the transcriptional level of pIgR. The salivary gland epithelial cells of the 60 patients with oral bacterial infection were isolated and extracted, and they were divided into two groups (observation group and control group) randomly. Agonists were added to the observation group for acting for 24 h. FQ-PCR and immunofluorescence (IF) were adopted to detect and compare the transcriptional level of pIgR after acting with agonists. The toxicity of agonists on the cells was detected with Cell Counting kit-8 (CCK-8). The isolated salivary gland epithelial cells conformed to the morphology of epithelial cells, and adhered to the wall for growing. The transcriptional level of pIgR in the bacterial infection group was lower than that in the non-bacterial infection group $(p<0.05)$. The transcriptional level of pIgR in the observation group was higher than that in the control group $(\mathrm{p}<0.05)$ after acting with agonists. Agonists can promote the rise of transcriptional level of pIgR in salivary gland epithelial cells, and the increase in pIgR is closely related to the cure of oral bacterial infection. Therefore, agonists can improve the oral immune function by regulating the transcription of $\mathrm{pIgR}$.
\end{abstract}

Correspondence to: Dr Li Huang, Department of Stomatology, Renmin Hospital of Wuhan University, 99 Zhagnzhidong Road, Wuhan, Hubei 430060, P.R. China

E-mail: youbang0902@163.com

Key words: agonists, salivary gland epithelial cells, pIgR, transcription

\section{Introduction}

Oral cavity is a naturally open system, and the immune molecules in the saliva play important roles in immune defense and protection (1). The regulation of the flora in the mouth to keep them balanced, and the prevention of dental caries decide the oral health. The integrity of the mucosa should be maintained to avoid direct effects of harmful substances on the mucosa. The substances in the oral cavity should be cleared and excreted to keep the mouth relatively clean and sanitary, e.t.c. $(2,3)$. As an important member of mucosal immune, polymeric immunoglobulin receptor (pIgR) is a member of immunoglobulin superfamily, and the only transporting receptor of polymeric immunoglobulin A (pIgA) and polymeric immunoglobulin M (pIgM). It is synthesized from mucosa epithelial cells and exocrine gland epithelial cells, and can clear antigen and harmful substances in the mucosa, which plays an important role in the first line of defense in the immune system $(4,5)$. Therefore, the study on the active ingredient (pIgR) in the saliva is of great significance and prospect. This study analyzed the mechanism of agonists in regulating transcriptional level of pIgR in salivary gland epithelial cells, thus revealing the defense effect of salivary immune on bacteria in the oral cavity.

\section{Materials and methods}

General data. A total of 60 patients with oral bacterial infection and 70 patients suffering from oral diseases without bacterial infection were selected randomly from patients that were treated in Renmin Hospital of Wuhan University (Wuhan, China) from April 2015 to April 2017. Inclusion criteria: patients whose sublingual gland tissues needed to be removed for the treatment of their diseases. Exclusion criteria: patients with severe diseases in cardiovascular or digestive systems, mental diseases, infectious diseases, e.t.c., that had an impact on the test results. Patients in the group with bacterial infection of other organs were aged 19-51 years old with an average of 36.12 \pm 10.32 years old, including 38 males and 22 females, among which 22 patients were mainly infected with oral streptococci, 10 with Streptococcus salivarius and 28 with Streptococcus sanguis. Patients in the group without bacterial infection were aged 18-52 years old with an average of $35.78 \pm 11.42$ years old, including 44 males and 26 females. The data (such as age and sex) of the two groups of patients had 
no statistical differences ( $p>0.05)$, and they were comparable. The study was approved by the Ethics Committee of Renmin Hospital of Wuhan University and written informed consents were signed by the patients.

Reagents. DMEM/F12 culture medium (HyClone; GE Healthcare Life Sciences, Logan, UT, USA); fetal bovine serum (FBS) (Lanzhou Bailing, Lanzhou, China); TRIzol (Life Technologies; Thermo Fisher Scientific, Inc., Waltham, MA, USA); reverse transcription kit (Takara Biotechnology Co., Ltd., Dalian, China); SYBR ${ }^{\circledR}$ Premix Ex Taq ${ }^{\mathrm{TM}}$ II kit (Takara Biotechnology Co., Ltd.); rabbit anti-human $\mathrm{pIgR}$ primary antibody and goat anti-rabbit secondary antibody (Abcam, Cambridge, MA, USA); cellulose acetate membrane (EMD Millipore, Burlington, MA, USA); developing liquid and fixing liquid (Beijing Transgen Biotech Co., Ltd., Beijing, China), internal reference glyceraldehyde-3-phosphate dehydrogenase (GAPDH), primer of pIgR (Shanghai Sangon, Shanghai, China), and Cell Counting kit-8 (CCK-8) (Dojindo Molecular Technologies, Inc., Shanghai, China).

\section{Methods}

Isolation of salivary gland epithelial cells (6). The removed salivary glands were washed with sterile phosphate-buffered saline (PBS) for 2-3 times. They were cut into small pieces $\left(1-2 \mathrm{~mm}^{3}\right)$ with a scissor, and incubated in the culture plates. Dulbecco's modified Eagle's medium/F12 (DMEM/F12) [containing penicillin, streptomycin, epidermal growth factor (EGF), insulin and hydrocortisone] with 3\% FBS was added. Then they were placed in the incubator containing 5\% carbon dioxide $\left(\mathrm{CO}_{2}\right)$ for culturing. Three days later, the culture solution was replaced. Thereafter, the culture solution was replaced once every 3 days. The cells were passaged when $70-80 \%$ cells grew and fused.

Fluorescent quantitative polymerase chain reaction (FQ-PCR) analysis on transcriptional level of pIgR. Ribonucleic acid (RNA) of the cell specimen was extracted, and the concentration was measured. RNA $(1 \mu \mathrm{g})$ was taken to carry out reverse transcription reaction with reverse transcription enzyme-reagent kits, thus obtaining complementary DNA (cDNA). The concentration of cDNA was adjusted, and Bio-Rad CFX 96 PCR (Bio-Rad Laboratories, Inc., Hercules, CA, USA) was used to measure the relative expression volume of messenger RNA (mRNA) of different groups according to the illustration of $\mathrm{SYBR}^{\circledR}$ Premix Ex Taq ${ }^{\mathrm{TM}}$ II kits. The sequences of the corresponding primers are shown in Table I, and the setting of PCR program is demonstrated in Table II.

Detection of protein expression of pIgR with western blotting $(7,8)$. Salivary gland cells were digested with pancreatin and transferred to the radio-immunoprecipitation assay (RIPA) protein lysate. The resulted solution was lysed for $30 \mathrm{~min}$ on ice, during which vortex mixing was conducted for 3 times. Then it was centrifuged for $10 \mathrm{~min}$ at $4^{\circ} \mathrm{C}$ in a rate of $12,000 \mathrm{x} \mathrm{g}$. The supernatant was filled in a $1.5 \mathrm{ml}$ Eppendorf (EP) tube. Part of the supernate was taken to detect the concentration of protein by bicinchoninic acid (BCA) method. Then part of the supernatant was taken to ensure that the content of protein in each specimen waiting for test was
Table I. Primer sequences of GAPDH, alkaline phosphatase (ALP) and osteocalcin (OCN).

Name of

the gene

Sequence of primers

$\beta$-actin Forward: 5'-GCTTGGAATGAGACTGCTGA-3' Reverse: 5'-CTGGCCATATCCACCAGAGT-3'

pIgR Forward: 5'-TCAGGTGCTTTGCTAGATG-3' Reverse: 5'-TTTGGGTGTAAGAATGGTAA-3'

Table II. Program of PCR.

\begin{tabular}{lccr}
\hline Step & Temperature & Time & Circulation \\
\hline 1 & $94^{\circ} \mathrm{C}$ & $15 \mathrm{~min}$ & 1 \\
2 & $94^{\circ} \mathrm{C}$ & $10 \mathrm{sec}$ & 40 \\
& $50^{\circ} \mathrm{C}$ & $30 \mathrm{sec}$ & \\
& $72^{\circ} \mathrm{C}$ & $15 \mathrm{sec}$ & \\
& & Read & \\
3 & $72^{\circ} \mathrm{C}$ & $10 \mathrm{~min}$ & 1 \\
\hline
\end{tabular}

$100 \mu \mathrm{g}$. Reduced loading buffer (5X) was added, and boiled with boiled water for $10 \mathrm{~min}$. The afore-mentioned sample solution was added slowly to the prepared gel wells for sample loading with microsyringes. Sodium dodecyl sulfate polyacrylamide gel electrophoresis (SDS-PAGE; 15\%) was conducted under the voltage of $80 \mathrm{~V}$. Wet transfer was conducted for $0.5 \mathrm{~h}$ under the voltage of $40 \mathrm{~V}$ after the end of electrophoresis. The target protein in the gel was transferred to nitrocellulose (NC) membrane. Then the membrane was rinsed with eluent for at least 3 times (10 min/time), and rabbit anti-human pIgR polyclonal antibody (1:600; cat no. ab96196; Abcam) was added to the membrane. The membrane was blocked overnight with skimmed milk powder at $4^{\circ} \mathrm{C}$. The primary antibody was incubated for $2 \mathrm{~h}$, and the goat anti-rabbit secondary polyclonal antibody (1:1,000; cat no. ab6721; Abcam) was incubated for $1 \mathrm{~h}$ at room temperature. Fluorogenic substrate was added, and pressed in the dark room for the formation of images.

Detection of protein expression in $\mathrm{pIg} R$ with immunofluorescence (9). The slides filled with cells in the culture plates were rinsed with PBS for 3 times ( 3 min/time). The slides were fixed with $4 \%$ paraformaldehyde for $15 \mathrm{~min}$, and rinsed with PBS for 3 times ( $3 \mathrm{~min} /$ time). Triton X-100 (0.5\%) (prepared with PBS) was added, and allowed to stand at room temperature for $20 \mathrm{~min}$. The glass slides were rinsed with PBS for 3 times ( $3 \mathrm{~min} / \mathrm{time}$ ); then absorbent paper was used to absorb PBS. Normal goat serum was dripped on the glass slides, and they were blocked at room temperature for $30 \mathrm{~min}$. A sufficient amount of diluted primary antibody was dripped to each slide. Then the slides were incubated in the wet box overnight at $4^{\circ} \mathrm{C}$. Fluorescent secondary antibody was added. The slides were rinsed with PBS containing Tween-20 (PBST) for 3 times (30 $\mathrm{min} / \mathrm{time}$ ), and incubated in the wet box at room 


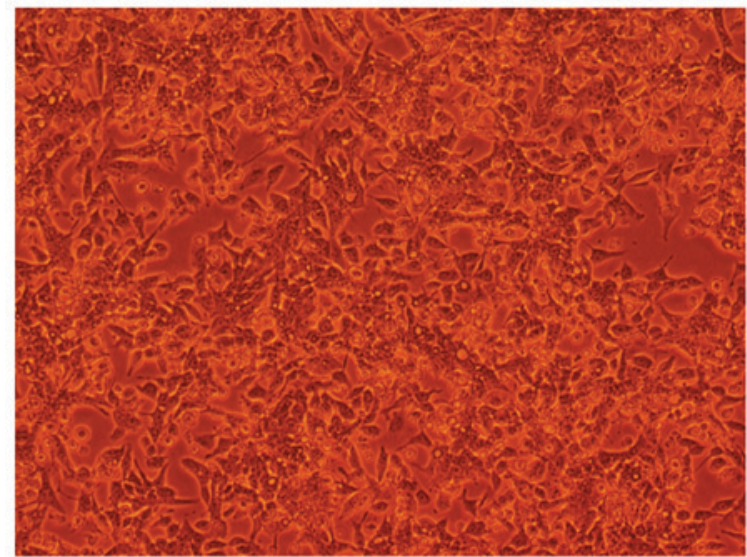

Figure 1. Salivary gland epithelial cells that were cultured in vitro by adhering to the wall.

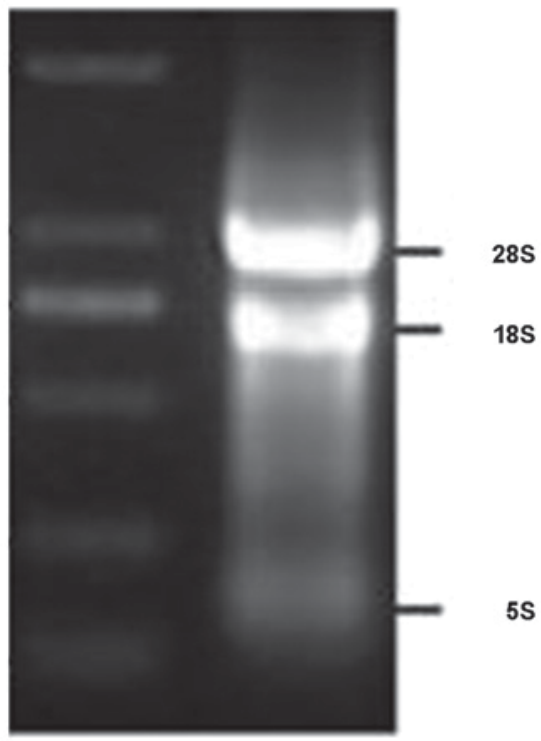

Figure 2. Agarose gel electrophoresis of RNA.

temperature for $1 \mathrm{~h}$. They were rinsed with PBST, and cut into slices for 3 times ( $3 \mathrm{~min} / \mathrm{time}$ ). Diamidino-phenyl-indole (DAPI) was added, and the slides were incubated away from light for $5 \mathrm{~min}$. Staining was conducted for the specimen, and the slides were rinsed with PBST to remove excessive DAPI. The slides were sealed with slide-sealing solution containing anti-fluorescence quenching agent; then the slides were placed under the fluorescence microscope to observe and collect the images.

Detection of survival rate of cells with CCK-8. Cell suspension $(100 \mu \mathrm{l})$ was prepared in 96-well plates. All the blank group, observation group and control group had 3 repeated wells. The culture plates were cultured in the incubator until the density of the cells reached 50\%. Agonist solution (10 $\mu \mathrm{l})$ was added to the observation group, and the equivalent volume of culture medium was added to the control group for incubation. Twenty-four hours later, $10 \mu \mathrm{l}$ of CCK-8 solution was added to each well, and they were placed in the incubator for $2 \mathrm{~h}$. The optical density at $450 \mathrm{~nm}$ was detected with microplate reader.
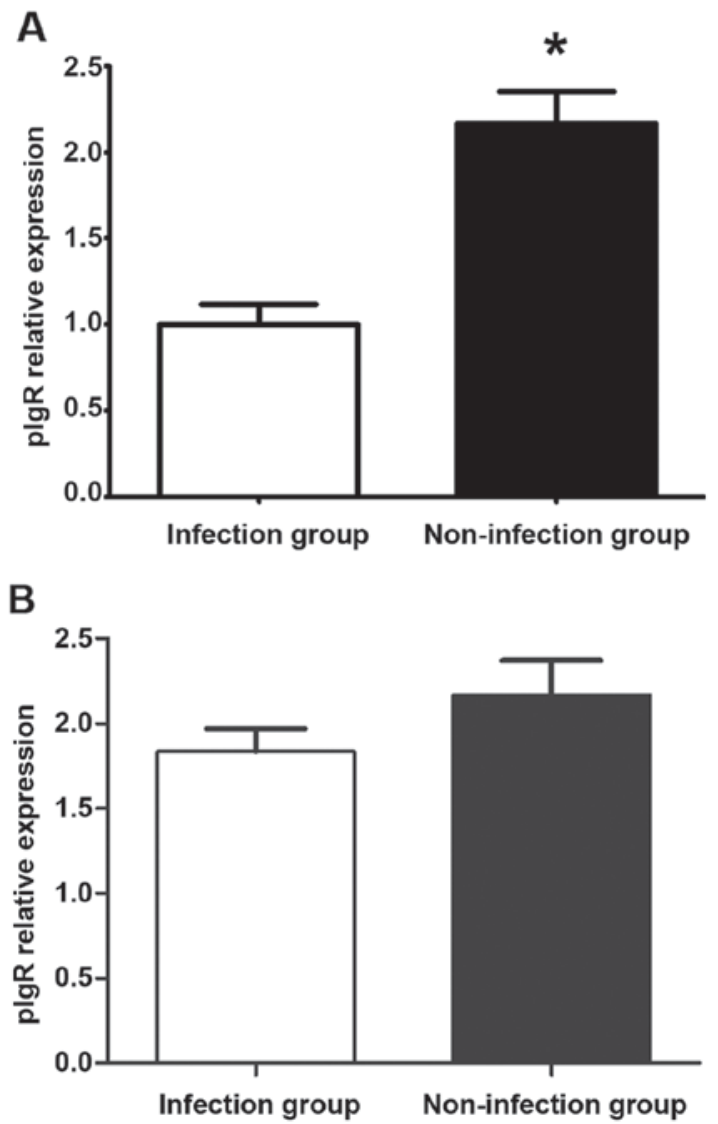

Figure 3. Results of FQ-PCR of the two groups (A) before and (B) after treatment. Relative expression level of pIgR in the bacterial infection group is lower than that in the non-infection group before treatment $\left({ }^{*} \mathrm{p}<0.05\right)$, while the relative expression level of $\mathrm{pIgR}$ in the bacterial infection group is increased after treatment, and the difference with that in the non-infection group has no statistical significance $(\mathrm{p}>0.05)$.

Survival rate of cells $=(\mathrm{OD}$ in the observation group - OD in the blank group) / (OD in the control group - OD in the blank group).

Statistical methods. Statistical treatment was carried out with Statistical Product and Service Solutions (SPSS, Inc., Chicago, IL, USA) 17.0 software, and the test data were expressed as $($ mean $\pm \mathrm{SD}$ ). t-test was used for comparison. $\mathrm{P}<0.05$ suggested that the comparison had a statistical difference.

\section{Results}

Isolation of salivary gland epithelial cells. The tissue blocks of salivary gland epithelial cells adhered to the wall for growing. They lined up closely and showed a typical shape of 'paving stones'. It could be seen that small round and polygonal cells inlaid each other (Fig. 1).

Comparison of transcriptional level of pIgR in the infection group with that in the non-infection group before and after treatment. Total RNA of salivary gland epithelial cells with the purity (A260/A280) of 2.02 was extracted (Fig. 2). The results of FQ-PRC and western blotting methods showed that the transcriptional level of pIgR in the bacterial infection group was lower than that in the non-infection group before 
A

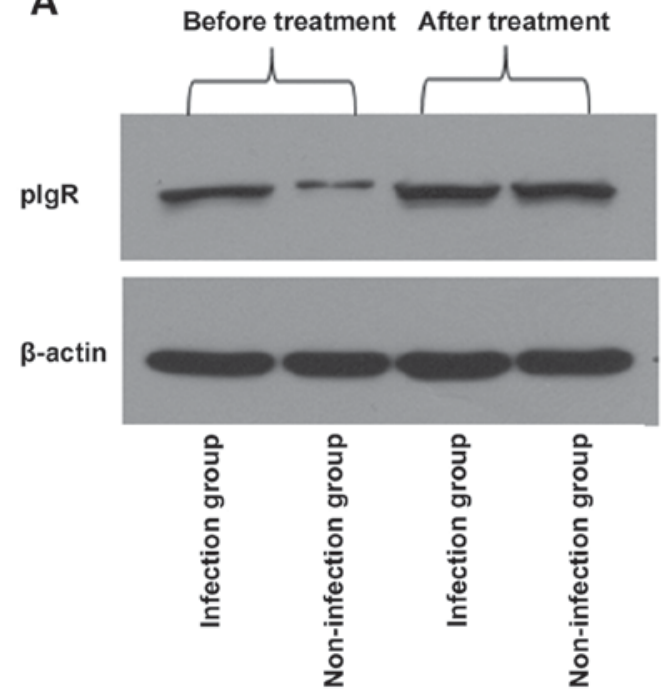

B

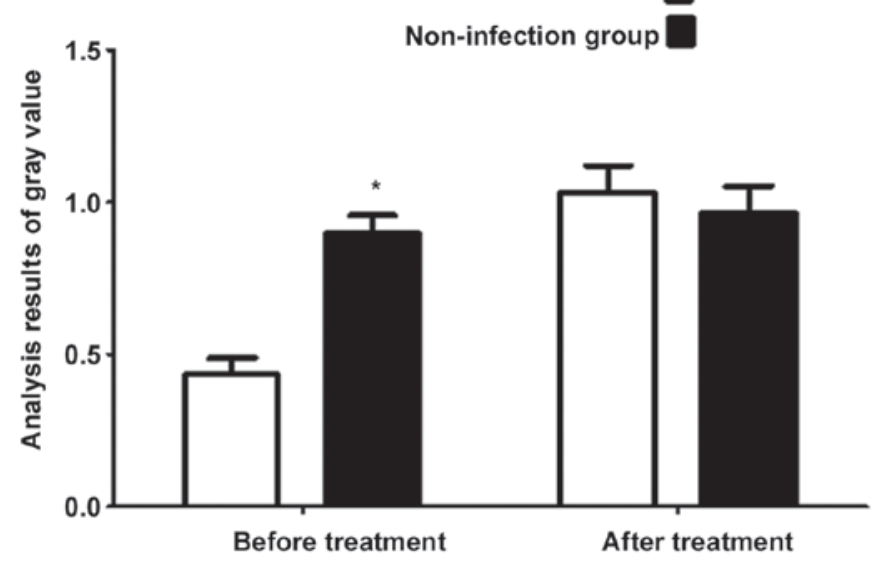

Figure 4. Protein expression before and after treatment. Relative expression level of pIgR in the bacterial infection group is lower than that in the non-infection group before treatment $\left({ }^{*} \mathrm{p}<0.05\right)$, while the relative expression level of pIgR in the bacterial infection group is increased after treatment, and the comparison with that in the non-infection group has no statistical significance ( $\mathrm{p}>0.05$ ). (A) Western blotting results of the two groups before and after treatment. (B) Analysis results of gray level of protein expression in the two groups before and after treatment.

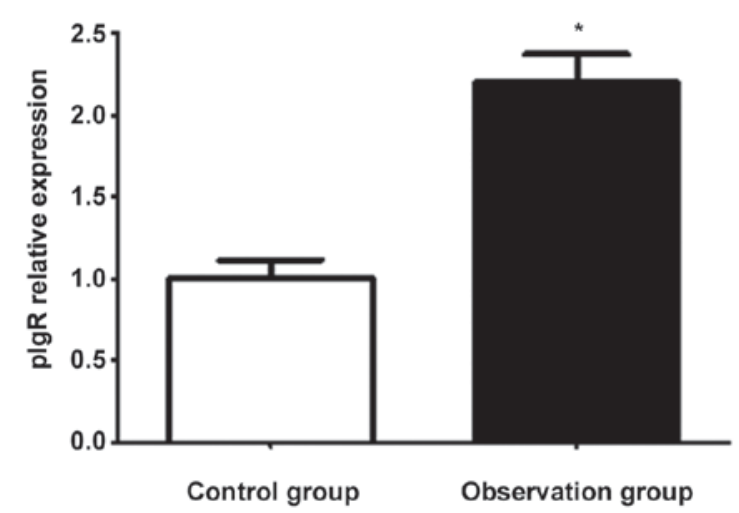

Figure 5. FQ-PCR results show that the transcriptional level of pIgR in the observation group is higher than that in the control group ( $\mathrm{p} p<0.05)$.

treatment $(p<0.05)$, while the transcriptional level of $\mathrm{pIgR}$ in the bacterial infection group was increased after treatment, and the difference with that in the non-infection group showed no statistical significance ( $\mathrm{p}>0.05$ ) (Figs. 3 and 4).

Impacts of agonists on transcription of pIgR in salivary gland epithelial cells. FQ-PCR results showed that the transcriptional level of $\mathrm{pIgR}$ in the observation group was higher than that in the control group $(\mathrm{p}<0.05)$ after acting with agonists (Fig. 5). The immunofluorescence results indicated that the protein expressed by transcription in the observation group was higher than that in the control group (Fig. 6).

Detection of toxicity of agonists on the cells with CCK-8. The average OD of blank wells was 0.212 , and that in the control group and the observation group was 0.4114 and 0.402 , respectively. It was calculated that the survival rate of cells in the observation group was $95.9 \%$, while that in the control group was $100 \%$. The difference had no statistical significance $(\mathrm{p}>0.05)$.

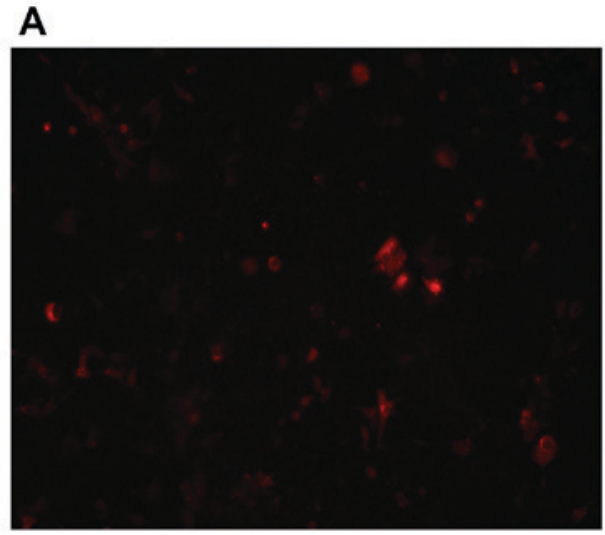

\section{B}

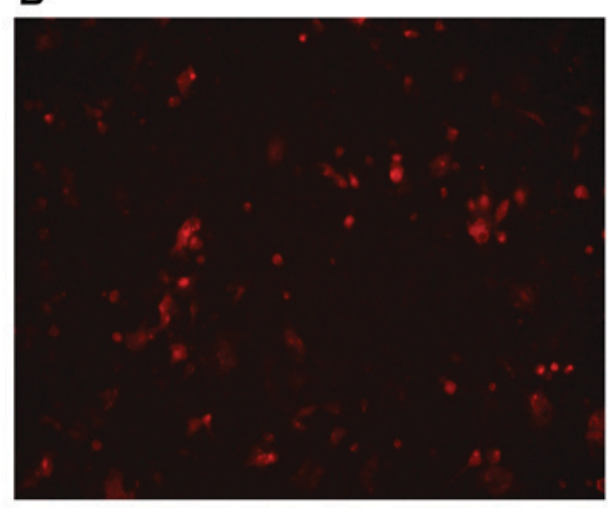

Figure 6. The immunofluorescence results indicate that the protein expressed by transcription in the observation group is higher than that in the control group. (A) Immunofluorescence result of the control group. (B) Immunofluorescence result of the observation group.

\section{Discussion}

There is a large number of microbial communities in the oral cavity, and in a sense, the health of the oral cavity is a 
reflection of the ecological balance of the bacteria. Once the balance is broken, the beneficial bacteria will be reduced, which will result in all kinds of oral inflammation and diseases $(10,11)$. Oral streptococcus that often lives in the oral cavity plays an important role in the oral micro-ecological system, among which Streptococcus mutans are the main pathogenic bacteria of caries, Streptococcus sanguis is the pioneer in dental plaque, and Streptococcus buccalis and Streptococcus salivarius are the early colonized bacteria of oral mucosa (12). Colonization and dissemination of oral microorganisms are regulated and defended by the salivary immune system (13). Saliva is an important substance in oral immunity, and it contains lysozyme. There are lymphocytes and plasmacytes in the mesenchyme of salivary glands. IgA secreted by plasmacytes binds to the protein secretory components secreted by glandular cells, thus forming secreted IgA, which is released into the oral cavity with the saliva, and has an immunogenic effect (14). In recent years, the discussion on the effects of Streptococcus salivarius and Streptococcus mutans from the aspect of immunity has been a hotspot of research. A study of Carrasco-Yepez et al (15) found that the effects of Streptococcus salivarius and Streptococcus mutans are not specific. They are a manifestation of broad-spectrum non-specific effects. Kortum et al (16) held the opinion that alkaline phosphatase, lactate dehydrogenase and other relevant components in saliva immunity are highly correlated with the incidence of dental caries.

pIgR mediates the transcription of polymeric immunoglobulin in the epithelial cells, and participates in the formation of secretory immunoglobulin A (S-IgA) (17). pIgR has high affinity with dimer IgA (dIgA) with J chain near the external side of the base of the lamina propria, and forms a complex of ligand and receptor. Then the complex is actively transported to the apical end of the cell from the external side of the base through intracellular transport. The extracellular segment of pIgR binds covalently to $\mathrm{dIgA}$ at the apical end of the cell, and cleaves between transmembrane region and extracellular segment, thus secreting S-IgA. When viruses or foreign antigens penetrate the mucosal surface, and enter the lamina propria, pIgR also binds to immune complexes formed by $\operatorname{IgA}$ and antigen (Ag) to expel pathogens such as viruses and bacteria out of the body through transcytosis (18). A study conducted by DeSantis et al (19) showed that the impacts of $\mathrm{pIgR}$ on the structure, expression distribution, function and expression regulation are significantly associated with fish mucosal immunity. Qin et al (20) believed that polyimmunoglobulin receptors and secretion components are involved in multiple molecular mechanisms, and they play a very important role in mucosal immunity. The results of this study also found that the transcriptional expression of $\mathrm{pIgR}$ in salivary gland epithelial cells had a great relationship with diseasetreating streptococcus in the oral cavity. The transcriptional level of $\mathrm{pIgR}$ in the bacterial infection group was lower than that in the non-infection group before treatment, while the transcriptional level of $\mathrm{pIgR}$ in the bacterial infection group was increased after treatment, and the level was equivalent to that in the non-infection group, which showed that oral bacterial infection can reduce the content of $\mathrm{pIgR}$ in oral cavity, which in turn leads to the decrease of oral salivary immunity. Agonist is a substance that enhances the transcriptional level of pIgR. This study also proved that transcriptional level of $\mathrm{pIgR}$ in the observation group was higher than that in the control group after acting with agonists $(\mathrm{p}<0.05)$, which suggested that promoters can affect the oral salivary immune function by improving the transcriptional level of $\mathrm{pIgR}$, thus preventing and treating pathogens such as bacteria and virus in the mouth. Moreover, the use of agonists has no influence on normal proliferation of salivary gland epithelial cells, and it has relatively high safety.

In conclusion, agonists can promote the rise of the transcriptional level of pIgR in salivary gland epithelial cells, and the increase in pIgR is closely related to the cure of oral bacterial infection. Therefore, agonists can improve the oral immune function by regulating the transcription of $\mathrm{pIgR}$.

\section{Acknowledgements}

Not applicable.

\section{Funding}

This study was sponsored by the National Natural Science Fund (81600860).

\section{Availability of data and materials}

The datasets used and/or analyzed during the current study are available from the corresponding author on reasonable request.

\section{Authors' contributions}

LH and CS assisted with PCR and western blotting. RP and ZL were responsible for immunofluorescence. All authors read and approved the final manuscript.

\section{Ethics approval and consent to participate}

The present study was approved by the Ethics Committee of Renmin Hospital of Wuhan University (Wuhan, China) and written informed consents were signed by the patients.

\section{Patient consent for publication}

Not applicable.

\section{Competing interests}

The authors declare that they have no competing interests.

\section{References}

1. Rombout JH, Yang G and Kiron V: Adaptive immune responses at mucosal surfaces of teleost fish. Fish Shellfish Immunol 40: 634-643, 2014

2. López MC: Chronic alcohol consumption regulates the expression of poly immunoglobulin receptor (pIgR) and secretory $\operatorname{IgA}$ in the gut. Toxicol Appl Pharmacol 333: 84-91, 2017.

3. Ward-Caviness CK, Neas LM, Blach C, Haynes CS, LaRocqueAbramson K, Grass E, Dowdy ZE, Devlin RB, Diaz-Sanchez D, Cascio WE, et al: A genome-wide trans-ethnic interaction study links the PIGR-FCAMR locus to coronary atherosclerosis via interactions between genetic variants and residential exposure to traffic. PLoS One 12: e0173880, 2017. 
4. Li D, Wang FJ, Yu L, Yao WR, Cui YF and Yang GB: Expression of pIgR in the tracheal mucosa of SHIV/SIV-infected rhesus macaques. Zool Res 38: 44-48, 2017.

5. Yue X, Ai J, Xu Y, Chen Y, Huang M, Yang X, Hu B, Zhang $\mathrm{H}, \mathrm{He} \mathrm{C}$, Yang $\mathrm{X}$, et al: Polymeric immunoglobulin receptor promotes tumor growth in hepatocellular carcinoma. Hepatology 65: 1948-1962, 2017.

6. Yang S, Liu S, Qu B, Dong Y and Zhang S: Identification of sea bass $\mathrm{pIgR}$ shows its interaction with vitellogenin inducing antibody-like activities in HEK 293T cells. Fish Shellfish Immunol 63: 394-404, 2017.

7. Armitage CW, O'Meara CP and Beagley KW: Chlamydial infection enhances expression of the polymeric immunoglobulin receptor (pIgR) and transcytosis of IgA. Am J Reprod Immunol 77: e12611, 2017.

8. Qi X, Li X and Sun X: Reduced expression of polymeric immunoglobulin receptor (pIgR) in nasopharyngeal carcinoma and its correlation with prognosis. Tumour Biol 37: 11099-11104, 2016.

9. Zhang F, Liu D, Wang L, Li T, Chang Q, An L and Yang G: Characterization of IgM-binding protein: A pIgR-like molecule expressed by intestinal epithelial cells in the common carp (Cyprinus carpio L.). Vet Immunol Immunopathol 167: 30-35, 2015.

10. Yamamoto Y, To M, Hayashi T, Shimizu T, Kamata Y, Saruta J, Takahashi $\mathrm{T}$ and Tsukinoki $\mathrm{K}$ : Intake of indigestible carbohydrates influences IgA response and polymeric Ig receptor expression in the rat submandibular gland. Br J Nutr 113: $1895-1902,2015$

11. Simpfendorfer KR, Strugnell RA, Brodnicki TC and Wijburg OL: Increased autoimmune diabetes in pIgR-deficient NOD mice is due to a 'Hitchhiking' interval that refines the genetic effect of Idd5.4. PLoS One 10: e0121979, 2015.

12. Sankineni S, Cho Y, Hosseinian N and Kolliputi N: Does pIgR down-regulation in COPD cause reprogramming of bronchia epithelium? Lung 193: 1-2, 2015.

13. Niu H, Wang K and Wang Y: Polymeric immunoglobulin receptor expression is predictive of poor prognosis in glioma patients. Int J Clin Exp Med 7: 2185-2190, 2014.
14. Iovino F, Molema G and Bijlsma JJ: Streptococcus pneumoniae Interacts with pIgR expressed by the brain microvascular endothelium but does not co-localize with PAF receptor. PLoS One 9: e97914, 2014

15. Carrasco-Yepez M, Campos-Rodriguez R, Lopez-Reyes I, Bonilla-Lemus P, Rodriguez-Cortes AY, Contis-Montes de Oca A, Jarillo-Luna A, Miliar-Garcia A and Rojas-Hernandez S: Intranasal coadministration of Cholera toxin with amoeba lysates modulates the secretion of IgA and IgG antibodies, production of cytokines and expression of pIgR in the nasal cavity of mice in the model of Naegleria fowleri meningoencephalitis. Exp Parasitol 145 (Suppl): S84-S92, 2014.

16. Kortum AN, Rodriguez-Nunez I, Yang J, Shim J, Runft D, O'Driscoll ML, Haire RN, Cannon JP, Turner PM, Litman RT, et al: Differential expression and ligand binding indicate alternative functions for zebrafish polymeric immunoglobulin receptor (pIgR) and a family of pIgR-like (PIGRL) proteins. Immunogenetics 66: 267-279, 2014

17. Trevisi P, Gandolfi G, Priori D, Messori S, Colombo M, Mazzoni M, Lallès JP and Bosi P: Age-related expression of the polymeric immunoglobulin receptor $(\mathrm{pIgR})$ in the gastric mucosa of young pigs. PLoS One 8: e81473, 2013.

18. Xu T, Xie W, Ma Y, Zhou S, Zhang L, Chen J, Cai M, Sun R, Zhang P, Yu S, et al: Leptin/OB-R pathway promotes IL-4 secretion from B lymphocytes and induces salivary gland epithelial cell apoptosis in Sjögren's syndrome. Oncotarget 8: 63417-63429, 2017.

19. DeSantis KA, Stabell AR, Spitzer DC, O'Keefe KJ, Nelson DA and Larsen M: RAR $\alpha$ and RAR $\gamma$ reciprocally control $\mathrm{K}^{+}$ progenitor cell expansion in developing salivary glands. Organogenesis 13: 125-140, 2017.

20. Qin R, Steel A and Fazel N: Oral mucosa biology and salivary biomarkers. Clin Dermatol 35: 477-483, 2017. 